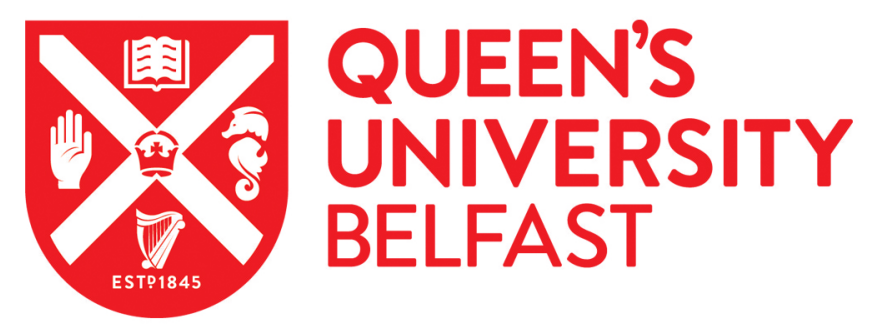

\title{
Muddy waters: efficacious predation of container-breeding mosquitoes by a newly-described calanoid copepod across differential water
} clarities

Cuthbert, R. N., Dalu, T., Wasserman, R. J., Coughlan, N. E., Callaghan, A., Weyl, O. L. F., \& Dick, J. T. A. (2018). Muddy waters: efficacious predation of container-breeding mosquitoes by a newly-described calanoid copepod across differential water clarities. Biological Control , 127, 25-30.

https://doi.org/10.1016/j.biocontrol.2018.08.017

Published in:

Biological Control

Document Version:

Peer reviewed version

Queen's University Belfast - Research Portal:

Link to publication record in Queen's University Belfast Research Portal

\author{
Publisher rights \\ Copyright 2018 Elsevier. \\ This manuscript is distributed under a Creative Commons Attribution-NonCommercial-NoDerivs License \\ (https://creativecommons.org/licenses/by-nc-nd/4.0/), which permits distribution and reproduction for non-commercial purposes, provided the \\ author and source are cited.
}

\section{General rights}

Copyright for the publications made accessible via the Queen's University Belfast Research Portal is retained by the author(s) and / or other copyright owners and it is a condition of accessing these publications that users recognise and abide by the legal requirements associated with these rights.

\section{Take down policy}

The Research Portal is Queen's institutional repository that provides access to Queen's research output. Every effort has been made to ensure that content in the Research Portal does not infringe any person's rights, or applicable UK laws. If you discover content in the Research Portal that you believe breaches copyright or violates any law, please contact openaccess@qub.ac.uk. 


\section{Muddy waters: efficacious predation of container-breeding mosquitoes by} a newly-described calanoid copepod across differential water clarities

Ross N. Cuthbert ${ }^{1,2,3^{*}}$, Tatenda Dalu ${ }^{4,5}$, Ryan J. Wasserman ${ }^{6,5}$, Neil. E. Coughlan $^{1}$, Amanda Callaghan ${ }^{3}$, Olaf L.F. Weyl ${ }^{2}$, Jaimie T.A. Dick ${ }^{1}$

${ }^{1}$ Institute for Global Food Security, School of Biological Sciences, Medical Biology Centre, (1) Queen's University Belfast, Belfast BT9 7BL, Northern Ireland Institute for Aquatic Biodiversity (SAIAB), Grahamstown 6140, South Africa

${ }^{3}$ Ecology and Evolutionary Biology, School of Biological Sciences, University of Reading, 0950, South Africa

${ }^{5}$ South African Institute for Aquatic Biodiversity (SAIAB), Grahamstown 6140, South Africa

${ }^{6}$ Department of Biological Sciences and Biotechnology, Botswana International University of Science and Technology, Palapye, Botswana

${ }^{*}$ Corresponding author: e-mail, rcuthbert03@qub.ac.uk; orcid ID, https://orcid.org/00000003-2770-254X 


\section{Abstract}

Mosquito-borne diseases induce unrivalled morbidity and mortality in human populations. In recent times, greater urbanisation has facilitated vector population expansion, particularly of those which proliferate in container-style habitats. To combat increased associated disease risk, we urgently require innovative and efficacious control mechanisms to be identified and implemented. Predatory biological control of vectorially-important mosquitoes can be effective. Despite their high prevalence in freshwater ecosystems, predatory calanoid copepods have yet to be examined comprehensively for mosquito control. Moreover, environmental context-dependencies can cause substantial variations in natural enemy impacts on target populations. Accordingly, improved understanding of the effects of context-dependencies upon predatory biocontrol is needed. Here, we use functional responses (FRs) to examine the predatory impact of a newly-described species of calanoid copepod, Lovenula raynerae, upon larval Culex pipiens prey across variations in prey supply and water clarity. Using outdoor field trials, we assess the viability of L. raynerae in reducing mosquito survival in container-style habitats. Lovenula raynerae displayed destabilising Type II FRs towards larval mosquito prey across all water clarities tested, with overall predation rates remaining largely unaffected across all clarity treatments. In the outdoor experiment, $L$. raynerae applications resulted in substantial reductions in larval C. pipiens populations, with close to total eradication achieved following the experimental period under higher predator densities. These results demonstrate that environmental context such as water clarity may have little effect on vector control by calanoid copepods, which suggests a predatory reliance on hydromechanical signalling. Further, for the first time, we demonstrate the applicability of calanoid copepods to artificial container-style habitats where mosquitoes proliferate.

Therefore, our results indicate that further examination into the applicability of this species group to aid vector biocontrol strategies is warranted. 


\section{$47 \quad$ Keywords}

biological control; predator-prey; functional response; turbidity; Culex pipiens; Lovenula raynerae

\section{Introduction}

The effective control of mosquito-borne diseases and their vectors is of substantial public health importance (Mehlhorn, 2012; Beneli and Mehlhorn, 2016; WHO, 2017). Currently, a variety of chemical, physical, genetic and biological approaches are used to control mosquitoes (see Becker et al. 2010). However, many population management approaches are associated with drawbacks which impede their sustainability (e.g.

Baldacchino et al. 2015). For instance, commonly-used insecticidal chemicals have caused environmental pollution, and emergent effects of insecticide resistance have presented major challenges to mosquito control strategies (e.g. Scholte et al. 2004; Ranson et al. 2016; Main et al. 2018). Mosquitoes which exploit artificial container-style habitats are of particular public health importance due to an association with urban areas and thus high potential for contact with human populations, wherein urban 'heat islands' can result in higher disease vector mosquito abundances (Townroe and Callaghan, 2014). Indeed, exploitation of human environments has facilitated invasive mosquito species to radically extend their geographic range (e.g. Lambrechts et al. 2010).

Biological control (hereafter biocontrol) provides a relatively environmentallyfriendly and economical option in vector control (Rodríguez-Pérez et al. 2012). Natural enemies can efficaciously suppress vectorially-important mosquito populations (Marten, 1990; Marten and Reid, 2007; Baldacchino et al. 2017; Cuthbert et al. 2018a, b; but see Thomas, 2018), and have successfully induced community-wide disease extirpations (Kay 
and Nam, 2005; Nam et al. 2012). However, many candidate biocontrol agents remain entirely unexplored, or underexploited in the context of container-style aquatic habitats where vectorially-efficient mosquitoes can proliferate en masse (e.g. Townroe and Callaghan, 2014). Biological control of larval mosquito populations by deliberate application of predatory copepod species has proven to be highly efficacious (reviewed by Marten and Reid, 2007). Presently, however, only those present within the cyclopoid order have been examined and utilised for control. Yet, copepods represent a vastly extensive group of crustaceans, comprising a broad range of orders adapted to both ephemeral and perennial hydrologic ecosystems (Dussart and Defaye, 2001). Despite previous erroneous categorisation as herbivorous, considered unable to prey upon mosquito larvae (Marten and Reid, 2007), predatory calanoid copepods exist and can exert profound trophic impacts in aquatic environments (Wasserman et al. 2016a; Dalu et al. 2016a; Cuthbert et al. 2018d). Moreover, certain calanoid copepod species can be atypically large in size (e.g. Suárez-Morales et al. 2015), and can therefore handle larval mosquito stages throughout their ontogeny (Cuthbert et al. 2018d). This contrasts to cyclopoid copepods which impart a size-refuge to larger prey (Marten and Reid 2007). Therefore, examining the efficacy of calanoid copepods towards container-breeding mosquitoes across ranging environmental contexts is of pertinence for the applied biocontrol of mosquito-borne disease vectors.

Environmental context-dependencies can cause substantial variations in natural enemy impacts on target populations (e.g. Cuthbert et al. 2018a), both as a result of biotic (e.g. Alexander et al. 2013; Barrios-O’Neill et al. 2014; Wasserman et al. 2016c) and abiotic (e.g. Wasserman et al. 2016b; Cuthbert et al. 2018a, b) factors, and is thus highly relevant to biocontrol agent selection. However, the implications of these context-dependencies on the efficacy of biocontrol agents often remain poorly understood. This, in turn, reduces the capacity of practitioners to fully understand and quantify biocontrol agent impacts. As 
vectorially-efficient mosquito species are adapted to breed in a highly variable range of aquatic habitats (see Becker et al. 2010), understanding the implications of environmental context is integral to robust quantifications of biocontrol agent impacts on target mosquito species. Further, finding biocontrol agents that are also robust to environmental variability would be desirable. In particular, water clarity is highly variable in hydrological environments, and variations in water clarity can affect food webs though alterations of predation efficacy by visual predators (e.g. van De Meutter et al. 2005; Lunt and Smee, 2015), manipulations of microhabitat structures and temperature regimes (e.g. Meysman et al. 2006; Paaijmans et al. 2017), and by directly impacting filter feeders (e.g. Rellstab and Spak, 2007), including larvae of many mosquito species. In addition, disease vector mosquitoes have been shown be attracted to low-clarity habitats due to perceived higher nutritional loads or greater depth (Ortiz-Perea and Callaghan, 2017; Cuthbert et al. 2018b), with concurrent implications for mosquito abundances (e.g. Medlock and Vaux, 2014). Therefore, identifying biocontrol agents to target disease vector mosquitoes which are not impacted by turbid environments is crucial for successful field applications in diverse aquatic habitats (see Cuthbert et al. 2018c).

Functional responses (FRs), i.e. the per capita consumption rates of consumers with changes to resource densities (Solomon, 1949; Holling, 1959; Juliano, 2001), have been applied extensively to quantify the resource regulation potential of consumers (e.g. Abrams, 1990; Dick et al. 2014), and can be applied to concurrently test environmental contextdependencies of consumer impact (e.g. South et al. 2017; Cuthbert et al. 2018a, b). Given that density- and context- dependencies of per capita impact may affect the viability of biocontrol agents in regulating target organisms (O’Neil, 1990; Van Driesche and Bellows, 1996; Cuthbert et al. 2018a), and the regulatory efficacy of many agents is yet to be explored, here, we examine the predatory potential of Lovenula raynerae Suárez-Morales, Wasserman and 
Dalu 2015, a recently described and remarkably large $(4-5 \mathrm{~mm})$ calanoid copepod species,

122

123

124 towards larvae of the disease vector complex Culex pipiens in container-style environments. Lovenula raynerae is a predatory ephemeral pond specialist species which hatches from dormant eggs within sediment during the early stages of hydroperiod (Suárez-Morales et al. 2015; Wasserman et al. 2016a). Such ephemeral aquatic systems are highly varied with respect to their water clarity, particularly as a result of bioturbation which can heavily impact ecosystem functioning (e.g. Waterkeyn et al. 2016). Although high predatory impacts of $L$. raynerae have recently been described upon larval mosquitoes across their ontogeny (Cuthbert et al. 2018d), further research is required to elucidate additional contextdependencies of their impact, alongside assessments of their use in container-style habitats which foster disease vector mosquitoes (Townroe and Callaghan 2014). Therefore, the present study examines the FRs of L. raynerae towards larvae of the mosquito C. pipiens across a water clarity gradient, and also assesses the predation potential of the copepod in outdoor artificial container-style habitats under varying modes of predator and prey density.

\section{Materials and methods}

\subsection{Animal collection and rearing}

Adult male and female L. raynerae $(4-5 \mathrm{~mm})$ were collected from an ephemeral pond in the Eastern Cape, South Africa ( $33^{\circ} 10^{\prime} 04.1^{\prime \prime} \mathrm{S} 27^{\circ} 16^{\prime} 10.6^{\prime \prime}$ E) by towing a $64 \mu \mathrm{m}$ zooplankton net through the upper water column. Copepods were transported in source water to a controlled environment $(\mathrm{CE})$ room at Rhodes University, Grahamstown $\left(25^{\circ} \mathrm{C} \pm 1{ }^{\circ} \mathrm{C}\right.$; 14:10 light:dark) and housed in $25 \mathrm{~L}$ aquaria containing strained $(200 \mu \mathrm{m})$ water from the collection site prior to the experiments. Culex pipiens complex larvae originated from egg rafts collected from artificial container-style aquatic habitats on the Rhodes University 
campus, and were reared to the desired size class on a diet of crushed rabbit food pellets

(Agricol, Port Eizabeth).

\subsection{Experimental protocols}

We conducted two experiments to discern the efficacy of the calanoid copepod $L$. raynerae in mosquito control. In experiment 1 , in the CE room, we quantified the effect of a water clarity gradient on the predatory impact of L. raynerae towards larval mosquito prey. Adult male L. raynerae were starved for $48 \mathrm{~h}$ prior to experimentation. Here, males were selected for experimentation to provide standardisation of predator type, given the various reproductive stages of female copepods that may influence predation rates. Functional responses of copepods were constrained under three water clarity treatments, conducive with the variability observed in ephemeral systems (Cuthbert, pers. obs.). Water clarity was defined as $0 \%, 50 \%$ and $100 \%$ against a predefined scale using a water clarity tube (GroundTruth, Leonard) by diluting turbid water to the prescribed clarity, with each treatment continuously aerated and filtered $(200 \mu \mathrm{m})$ prior to use. Culex pipiens $(3.3 \pm 0.2 \mathrm{~mm})$ larvae were established at five prey densities $(2,4,8,16,32 ; n=4$ per density) in $80 \mathrm{~mL}$ arenas of $5.6 \mathrm{~cm}$ diameter containing the appropriate clarity treatment. Once predators were added, they were allowed to feed undisturbed for $6 \mathrm{~h}$, after which they were removed and remaining prey counted to derive those killed. Controls consisted of three replicates at each density and clarity treatment without predators.

In experiment 2, we ascertained the efficacy of L. raynerae in outdoor artificial container-style habitats at regulating $C$. pipiens populations. This was done in a partially shaded outdoor location within the Rhodes University campus, similar to the environments from which C. pipiens rafts were collected. Culex pipiens larvae $(1.89 \mathrm{~mm} \pm 0.08)$ were added at two densities $(50,100)$, to $2 \mathrm{~L}$ arenas of $13.5 \mathrm{~cm}$ diameter, each containing $1.5 \mathrm{~L}$ 
filtered $(200 \mu \mathrm{m})$ aerated water from the copepod collection site and $0.3 \mathrm{~g}$ of crushed rabbit

169

170 food pellets. Then, three predator densities were added $(0,4,8)$ in a fully randomised array. We maintained copepod sex ratios of 3:1 male:female across predator treatments (i.e. 0:0, 3:1, 6:2) to minimise cannibalism (Lavens and Sorgeloos, 1996). After $72 \mathrm{~h}$, the predators were removed and remaining live mosquito prey counted to derive the number eaten. We conducted at least three replicates per experimental group. Water temperatures within arenas were found to be within the $16-20{ }^{\circ} \mathrm{C}$ range across the duration of the experiment.

\subsection{Statistical analyses}

All statistical analyses were undertaken in R v3.4.2. (R Core Team, 2017). In experiment 1, generalised linear models (GLMs) assuming a Poisson error distribution were used to examine the effects of 'water clarity' and 'prey density' on raw prey consumption. All FR analyses were undertaken within the 'frair' package in R (Pritchard et al. 2017). Logistic regression considering the proportion of prey consumed as a function of the 'prey density' factor was used to infer FR types. Here, a Type II FR is determined categorically by a significantly negative first order term, and a Type III FR by a significantly positive first order term followed by a significantly negative second order term. We fit Rogers' random predator equation to account for non-replacement of prey during the experiment (Trexler et al. 1998; Juliano, 2001):

$$
N_{e}=N_{0}\left(1-\exp \left(a\left(N_{e} h-T\right)\right)\right)
$$

where $N_{e}$ is the number of prey eaten, $N_{0}$ is the initial density of prey, $a$ is the attack constant, $h$ is the handling time and $T$ is the total experimental period. We applied the Lambert W function to fit the random predator equation (Bolker, 2008). The difference (delta) method 
(see Juliano, 2001) was employed to compare FR attack rates and handling times between treatments with respect to the 'water clarity' factor. We applied Bonferroni corrections to account for multiplicity of comparisons (i.e. $\alpha=0.017$ ). Furthermore, we employed a nonparametric bootstrapping procedure $(n=2000)$ to generate $95 \%$ confidence intervals around the FR curves (see Pritchard et al. 2017).

In experiment 2, GLMs assuming a quasibinomial error distribution, as residuals were found to be over-dispersed relative to degrees of freedom, were used to model mortality rates with respect to the 'predator density' and 'prey density' factors. Here, we used Tukey's comparisons via the 'multcomp' package in R (Hothorn et al. 2008). In all cases, nonsignificant terms and interactions were removed stepwise to obtain models with maximal parsimony (as per Crawley, 2007).

\section{Results}

In experiment 1 , survival in control groups was $100 \%$ and so experimental deaths of larval mosquitoes were attributed to predation by copepods, which were also observed eating the larvae. Overall consumption was not significantly affected by water clarity $\left(\chi^{2}=1.76, d f\right.$ $=2, p=0.42)$ but increased significantly with higher prey densities $\left(\chi^{2}=80.45, d f=4, p<\right.$ 0.001). The consumptive effect of 'prey density' was not dependent on the water clarity as the 'water clarity $\times$ prey density' effect was not significant $\left(\chi^{2}=7.46, d f=8, p=0.49\right)$. Type II FRs were detected in all water clarity treatments (Table 1; Figure 1). Functional response parameters (attack rate, $a$; handling time, $h$ ) did not differ significantly between any water clarity treatment pairs (Table 1 ; Figure $1 ; a$, low - medium, $z=0.64, p=0.52 ; a$, medium high, $z=0.25, p=0.80 ; a$, low - high, $z=0.87, p=0.39 ; h$, low - medium, $z=0.55, p=$ 0.58; $h$, medium - high, $z=0.79, p=0.43 ; h$, low - high, $z=0.32, p=0.75)$. 

mosquito reductions given that the 'predator density' factor significantly affected mortality rates $\left(F_{2,17}=72.59, p<0.001 ;\right.$ Figure 1$)$. Greater mortality rates were found between all incremental predator density increases $(0-4, z=6.69, p<0.001 ; 4-8: z=4.35, p<0.001 ; 0$ $-8, z=9.90, p<0.001)$. Significantly greater mortality rates of larval mosquito prey were demonstrated under the lower prey density treatments overall $\left(F_{1,16}=10.23, p=0.006\right.$; Figure 2). There was no 'predator density $\times$ prey density' interaction $\left(F_{2,14}=1.37, p=0.29\right)$, and so the efficacy of L. raynerae at different densities was robust to treatment variations associated with prey density.

\section{Discussion}

Here, for the first time, we demonstrate high per capita predation potential of an ephemeral pond specialist calanoid copepod, L. raynerae, towards vector mosquito prey irrespective of water clarity regime. Equally, in outdoor trials, we show that this species can substantially reduce larval mosquito abundances in container-style habitats which frequently foster vectorially-efficient mosquito species (Townroe and Callaghan, 2014). Copepods are highly efficacious predatory biocontrol agents for disease vectoring mosquitoes (Marten, 1984; Marten and Reid, 2007; Cuthbert et al 2018a, b). Although biocontrol examinations have hitherto focused on cyclopoid copepods, other groups of copepods are also predatory and thus may be of value in biocontrol strategies (Wasserman et al. 2016a; Cuthbert et al. 2018d).

Functional responses of the calanoid copepod L. raynerae were not significantly affected by variations in water clarity, either in terms of form or magnitude. In a predation context, both FR form and magnitude are powerful predictors of the interaction strengths between predators and prey (Dick et al. 2014), and may be combined with predator 
population responses to holistically assess ecological impact (Dick et al. 2017; Cuthbert et al. 2018a, b). Three broad forms of FR have been defined (Hassell, 1978): the linear Type I, hyperbolic Type II and sigmoidal Type III. Whilst Type I FRs are mechanistically restricted to filter feeders (Jeschke et al. 2004), Type II FRs are conducive to high ecological impact as a result of high predation pressures at low prey densities (Dick et al. 2014). Accordingly, Type II FRs are particularly desirable in biocontrol contexts as they trend towards target prey eradications (Cuthbert et al. 2018a). However, Type III FRs are regarded as more stabilising, wherein there is a provisioning of low-density refugia for prey, driven, empirically, by processes such as prey switching (Hassell, 1978; Cuthbert et al. 2018e).

The present study observed Type II FRs regardless of water clarity regime. Therefore, L. raynerae is effectively able to locate, capture and handle prey at low densities, even in highly turbid conditions. These results are pertinent as ephemeral aquatic ecosystems, both natural and artificial, are highly varied with respect to their water clarity regime, for instance due to detritus inputs or bioturbation (Cuthbert, pers. obs.). Indeed, bioturbation associated with biocontrol agents can also affect the viability of multiple management interventions in aquatic habitats which target larval mosquitoes (e.g. Fry-O’Brien and Mulla, 1996). Mosquitoes often exhibit predator avoidance behaviours when ovipositing (see Vonesh and Blaustein, 2010), and water clarity can offset this avoidance behaviour under certain conditions (Cuthbert et al. 2018b). For this reason, identifying predatory agents which are unaffected by water clarity variations is imperative for effective biocontrol applications. Our results suggest a reliance on hydromechanical cues by this predatory calanoid copepod when detecting and capturing mosquito prey, as opposed to visual cues, which may account for the lack of overall consumptive variation between water clarity treatments. Indeed, these results corroborate with those demonstrating a lack of reliance on visual signals in cyclopoid 
copepods when detecting prey across diurnal and water clarity regime shifts (e.g. Hwang and Strickler, 2001; Cuthbert et al. 2018c).

Attack rates of $L$. raynerae were relatively unaffected by variations in water clarity, and even trended towards being higher under lower water clarities. Attack rates correspond to the initial slope steepness in FR curves, and thus high attack rates can be particularly destabilising to prey populations at low prey densities. On the other hand, handling times reflect the asymptote in FR curves, and can be reciprocated to infer maximum feeding rates of predators (Dick et al. 2014; Cuthbert et al. 2018a). Here, although larval mosquitoes can exhibit high responsiveness to predatory cues (e.g. Zuharah and Lester 2011), it is feasible that low water clarity increases vulnerability to predation in larval mosquito prey, in turn enhancing the capture efficiency by predators at low prey densities. Furthermore, handling times were not significantly different across the water clarity gradient in the present study, and so maximum feeding rates were similar between treatments. Yet, handling times trended towards being lowest, and thus maximum feeding rates highest, at intermediate water clarities. Hence, we present strong and sustained destabilising predatory impacts of the calanoid copepod L. raynerae towards varying prey supplies of larval C. pipiens irrespective of this environmental context. Importantly, although FR examinations here only considered males, female L. raynerae are also voracious consumers of larval mosquitoes, with the potential to kill over 5 first instar larvae per hour and the ability to handle late instar prey (Cuthbert et al. 2018d). This intake rate is considerably higher than cyclopoid copepods which are often used in biological control, and which also impart a size refuge to late instar mosquito prey (Marten and Reid, 2007).

The study also highlights that the L. raynerae predation efficiency observed under controlled laboratory conditions also persists in outdoor environments exposed to natural conditions, where vector mosquitoes proliferate. In outdoor experiments within container- 
style habitats, L. raynerae induced substantial mortality rates in larval mosquito populations. As vectorially-important mosquitoes increasingly proliferate in such artificial, container-style habitats (Townroe and Callaghan, 2014), exploring the suitability for biocontrol agents over longer-term experiments in these environments is critical for empirical derivations of their efficacy. Indeed, these aquatic habitats can often be minute and ephemeral in nature, negating the use of larger larval mosquito antagonists, such as fish (see Azevedo-Santos et al. 2016). Our results demonstrate that, over the experimental period, higher densities of L. raynerae exhibited higher predation capacities relative to lower densities towards all prey supplies. Thus, multiple L. raynerae conspecifics may be additive in their consumption of C. pipiens larvae. Moreover, as L. raynerae is an ephemeral pond specialist and is capable of producing dormant, drought-resistant eggs, applications of this species to ephemeral aquatic habitats which foster mosquitoes may enable predator hatching in situ prior to, or simultaneous with, colonisation by mosquitoes. Although our results are theoretically promising in this respect, further research is required to test the efficacy of single applications of dormant eggs of $L$. raynerae over recurrent hydroperiods, and thus over longer experimental times overall. Furthermore, examinations of prey preferences and cannibalism in L. raynerae towards juveniles would be of value in further discerning factors that may impede their applied efficacy in biocontrol. However, it has been proposed that the most efficacious copepod species in biocontrol are able to curtail overpopulation and growth stunting via cannibalism of juveniles when the population becomes too high relative to the food supply (Marten and Reid, 2007).

In conclusion, our results suggest that calanoids and other copepod groups warrant further consideration as biocontrol agents of disease vector mosquitoes. In particular, ephemeral pond specialist species, such as L. raynerae, may be particularly promising candidates, as they are often particularly large, develop rapidly, exhibit dormancy, and have 
adapted to occupy relatively high trophic levels (Dalu et al. 2016a). Further, their especially

314 large size may make L. raynerae less vulnerable to higher-order predation as compared to

315

316

317 physically smaller copepod species. Generally, biotic interactions within ephemeral aquatic ecosystems are often poorly studied due to spatial and temporal heterogeneity (Dalu et al. 2016b), and thus these systems hold much potential for biocontrol agent exploration, as demonstrated in the present study. Our results show strong and destabilising predatory impacts of L. raynerae towards larvae of $C$. pipiens across a water clarity regime, which may enable consistently high impacts upon target populations under differing environmental conditions. Furthermore, we show efficacious predatory potential of this species in artificial container-style habitats in outdoor environments. Future research should test additional environmental contexts as to their effects on biotic interaction strengths between biocontrol agents and target organisms, and further explore the sustained potential of calanoid copepods over longer hydroperiods within container-style habitats which harbour disease vector mosquitoes.

\section{References}

Abrams, P.A., 1990. The effects of adaptive behaviour on the type-2 functional response. Ecology 71, 877-885.

Alexander, M.E., Dick, J.T.A., O’Connor, N.E., 2013. Trait-mediated indirect interactions in a marine intertidal system as quantified by functional responses. Oikos 122 , $1521-1531$.

Azevedo-Santos, V.M., Vitule, J.R.S., Pelicice, F.M., García-Berthou, E., Simberloff, D, 2017. Non-native fish to control Aedes mosquitoes: A controversial, harmful tool. BioScience 67, 84-90. 
Rizzoli, A. 2015. Control methods against invasive Aedes mosquitoes in Europe: a review.

Pest Manag. Sci. 71, 1471-1485.

Rizzoli, A., 2017. Predation efficiency of copepods against the new invasive mosquito

Alexander, M.E., Bovy, H.C., 2014. Fortune favours the bold: a higher predator reduces the impact of a native but not an invasive intermediate predator. J. Anim. Ecol. 83, 693-701. 2010. Mosquitoes and their control. Springer-Verlag, Berlin/Heidelberg. insights for mosquito vector control. Parasitol. Res. 115, 1747-1754. Princeton. agent selection under environmental change using functional responses, abundances and fecundities; the Relative Control Potential (RCP) metric. Biol. Control 121, 50-57. complexity and ovipositional responses modulate the efficacy of cyclopoid copepods in disease vector control. Biol. Control 121, 80-87. 

impact of cyclopoid copepods on larval mosquito Culex pipiens is unaffected by dyed environments. J. Vector Ecol. In press. 2018d. Calanoid copepods: an overlooked tool in the control of disease vector mosquitoes. J. Med. Entomol. In press.

Resistance is futile: lack of predator switching and a preference for native prey predict the success of an invasive prey species. Royal Soc. Open Sci. In press. interactions in an austral temperate ephemeral pond inferred using stable isotope analysis. Hydrobiologia 768, 81-94. drought frequency increases may have landscape-level consequences for ephemeral ecosystems. Glob. Change Biol. 23, 983-985.

Farnsworth, K.D., Richardson, D.M., 2014. Advancing impact prediction and hypothesis testing in invasion ecology using a comparative functional response approach. Biol. Invasions $16,735-753$. Médoc, V., Boets, P., Alexander, M.E., Taylor, N.G., Dunn, A.M., Hatcher, M.J., Rosewarne, P.J., Crookes, S., MacIsaac, H.J., Xu, M., Ricciardi, A., Wasserman, R.J., Ellender, B.R., 
382

383

384

385

386

387

Caffrey, J.M., 2017. Invader Relative Impact Potential: a new metric to understand and predict the ecological impacts of existing, emerging and future invasive alien species. J. Appl. Ecol. 54, 1259-1267.

Dussart, B.H., Defaye, D., 2001. Introduction to the Copepoda. Guides to the Identification of the Microinvertebrates of the Continental Waters of the World 16. Backhuys Publishers, Leiden.

Fry-O’Brien, L.L., Mulla, M.S., 1996. Effect of tadpole shrimp, Triops longicaudatus, (Notostraca: Triopsidae), on the efficacy of the microbial control agent Bacillus thuringiensis var. israelensis in experimental microcosms. J. Am. Mosq. Contr. Assoc. 12, 33-38. Hassell, M.P., 1978. The dynamics of arthropod predator-prey systems. Princeton University Press, Princeton.

Holling, C.S., 1959. Some characteristics of simple types of predation and parasitism. Can. Entomol. 91, 385-398.

Hothorn, T., Bretz, F., Westfall, P., 2008. Simultaneous inference in general parametric models. Biom. J. 50, 346-363.

Hwang, J.S., Strickler, R., 2001. Can copepods differentiate prey from predator hydromechanically? Zool. Stud. 40, 1-7

Jeschke, J.M., Kopp, M., Tollrian, R., 2004. Consumer-food systems: why type I functional responses are exclusive to filter feeders. Biol. Rev. 79, 337-349.

Juliano, S.A., 2001. Non-linear curve fitting: predation and functional response curves. In: Scheiner, S.M., Gurevitch, J. (Eds.), Design and Analysis of Ecological Experiments. Oxford University Press, Oxford, pp. 178-196. 

$365,613-617$. Lambrechts, L., Scott, T.W., Gubler, D.J., 2010. Consequences of the expanding 407 global distribution of Aedes albopictus for Dengue virus transmission. PLoS Negl. Trop. Dis. 408 $4,645$. aquaculture (No. 361). Food and Agriculture Organization (FAO), Rome.

Lunt, J., Smee, D.J., 2015. Turbidity interferes with foraging success of visual but not chemosensory predators. PeerJ 3, e1212. coluzzii. Parasit. Vectors 11, 225. green alga Kirchneriella irregularis upon larval Aedes albopictus (Diptera: Culicidae). Bull. Soc. Vector Ecol. 9, 1-5. Macrocyclops albidus (Copepoda, Cyclopidae). J. Am. Mosq. Control Assoc. 6, 689-693. $23,65-92$. 
427

428

429

430

431

432

and increase of expertise in arachno-entomology are urgently needed. Parasitol. Res. 110, $259-265$.

Meysman, F.J.R., Middelburg, J.J., Heip, C.H.R., 2006. Bioturbation: a fresh look at Darwin's last idea. Trends Ecol. Evol. 21, 688-695.

Nam, V.S., Yen, N.T., Duc, H.M., Tu, T.C., Thang, V.T., Le, N.H., Le Loan, L., Huong, V.T.Q., Khanh, L.H.K., Trang, H.T.T., Lam, L.Z.Y., Kutcher, S.C., Aaskov, J.G., Jeffery, J.A.L., Ryan, P.A., Kay, B.H., 2012. Community-based control of Aedes aegypti by using Mesocyclops in Southern Vietnam. Am. J. Trop. Med. Hyg. 86, 850-859.

O’Neil, R.J., 1990. Functional response of arthropod predators and its role in the biological control of insect pests in agricultural systems. In: Dunn, P.E., Baker, R.R. (Eds.), New Directions in Biological Control: Alternatives for Suppressing Agricultural Pests and Diseases. Alan R Liss Inc, New York, pp. 83-96.

Ortiz-Perea, N., Callaghan, A., 2017. Pond dyes are Culex mosquito oviposition attractants. PeerJ 5, e3361.

Paaijmans, K., Takken, W., Githeko, A.K., Jacobs, A.F.G., 2017. The effect of water turbidity on the near-surface water temperature of larval habitats of the malaria mosquito Anopheles gambiae. Int. J. Biomet. 52, 747-753.

Pritchard, D.W., Paterson, R.A., Bovy, H.C., Barrios-O’Neill, D., 2017. Frair: an R package for fitting and comparing consumer functional responses. Method. Ecol. Evol. 8, $1528-1534$.

R Core Team, 2017. R: A Language and Environment for Statistical Computing. R Foundation for Statistical Computing, Vienna. 
mosquitoes: a worsening situation that needs urgent action to maintain malaria control.

451 Trends Parasitol. 32, 187-96.

Rellstab C., Spaak P., 2007. Starving with a full gut? Effect of suspended particles on the fitness of Daphnia hyalina. Hydrobiol. 594, 131-139.

Control of Dengue Vectors. In: Larramendy, M.L. and Soloneski, S. (Eds.) Integrated Pest

functional responses of the dogfish Scyliorhinus canicula (Linnaeus, 1758) towards amphipod prey Echinogammarus marinus (Leach, 1815). Environ. Biol. Fish. 100, 12511563.

466 Schmeil (Copepoda, Diaptomidae) from the Eastern Cape province of South Africa.

467 Crustaceana 88, 324-342. 
471 of urbanisation and climate change on community composition and phenology. PLoS One 9, $472 \mathrm{e} 95325$.

Trexler, J.C., McCulloch, C.E., Travis, J., 1988. How can the functional response best 474 be determined? Oecologia 76, 206-214. prey and littoral predators: microhabitat selection by Daphnia induced by damselfly larvae.

477 Oikos 107, 265-272. selection: A meta-analysis and implications for vector control. Israel J. Ecol. Evol. 56, 123139.

Wasserman, R.J., Alexander, M.E., Barrios-O’Neill, D., Weyl, O.L.F., Dalu, T., 2016a. Using functional responses to assess predator hatching phenology implications for pioneering prey in arid temporary pools. J. Plankt. Res. 38, 154-158. 

impacts of the keystone predator Triops cancriformis (Branchiopoda: Notostraca) in temporary ponds. Freshwater Biol. 61, 1392-1404. http://www.who.int/mediacentre/factsheets/fs387/en/ (accessed 26 April 2018). predators? A test using native and exotic mosquito species in New Zealand. Popul. Ecol. 53, $307-317$.

\section{Acknowledgements}

500

501

502

503

504

This study forms part of a PhD studentship provided by the Department for the Economy, Northern Ireland. We extend gratitude to Rhodes University for the provision of laboratory facilities. We also thank Evans Mauda for assistance with the laboratory experiments. We acknowledge use of infrastructure and equipment provided by the SAIAB Research Platform and the funding channelled through the NRF-SAIAB Institutional Support system. This study was partially funded by the National Research Foundation - South African Research Chairs Initiative of the Department of Science and Technology (Inland Fisheries and Freshwater Ecology, Grant No. 110507). 
514 Table 1. First order terms generated from logistic regression of proportional prey

515 consumption as a function of prey density alongside parameter outputs from Rogers' random

516 predator equation across water clarity regimes.

\begin{tabular}{|l|l|l|l|}
\hline Water clarity & First order term, $p$ & $a, p$ & $h, p$ \\
\hline $0 \%$ & $-0.058620,<0.001$ & $1.572822,<0.001$ & $0.104547,<0.001$ \\
\hline $50 \%$ & $-0.044384,<0.001$ & $1.201939,<0.001$ & $0.087762,<0.001$ \\
\hline $100 \%$ & $-0.049398,<0.001$ & $1.082802,<0.001$ & $0.115452,<0.001$ \\
\hline
\end{tabular}

517

518

519

520

521

522

523

524

525

526

527 
528 Figure 1. Functional responses of Lovenula raynerae towards larval Culex pipiens prey

529 across a water clarity gradient (low, 0\%; medium, 50\%; high, 100\%). Shaded areas represent 530 bootstrapped $(n=2000)$ confidence intervals.

531 Figure 2. Mortality rate $( \pm \mathrm{SE})$ of larval Culex pipiens at different densities in outdoor trial

532 resulting from the presence of predatory copepod Lovenula raynerae at three densities.

533

534

535

536

537

538

539

540

541

542

543

544

545

546

547 

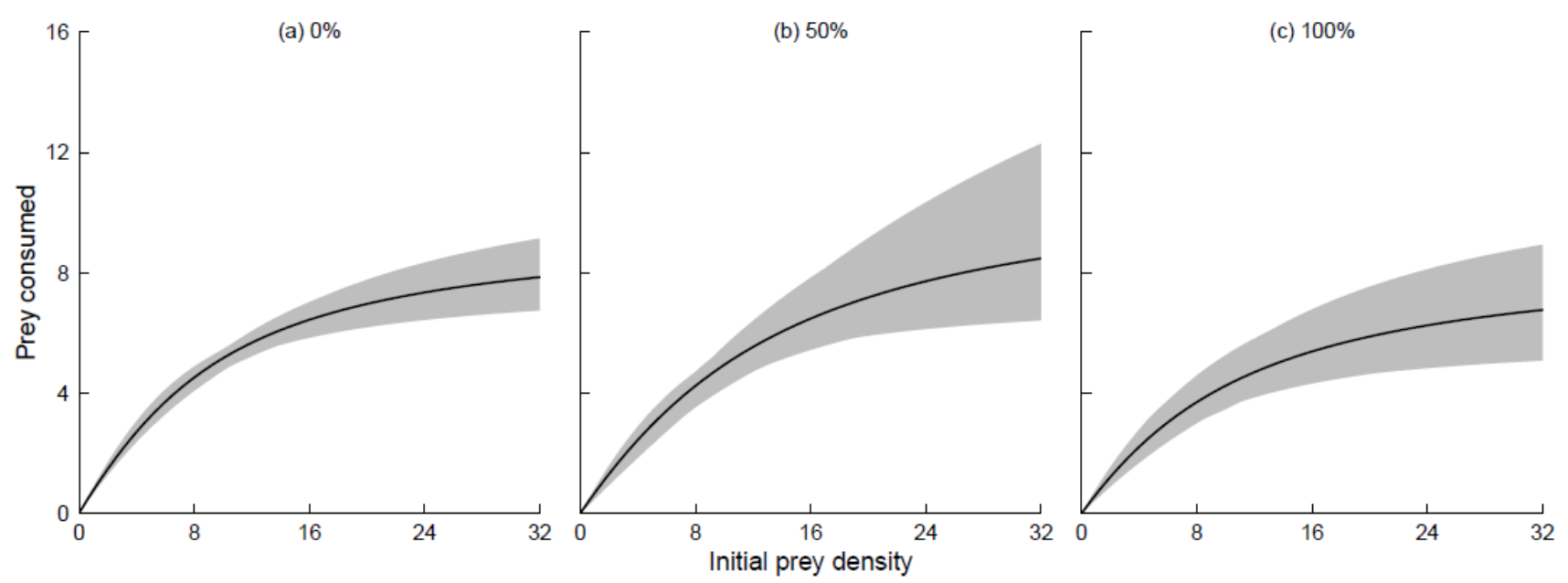

548

549

550

551

552

553

554

555

556

557

558

559

560

561

562 


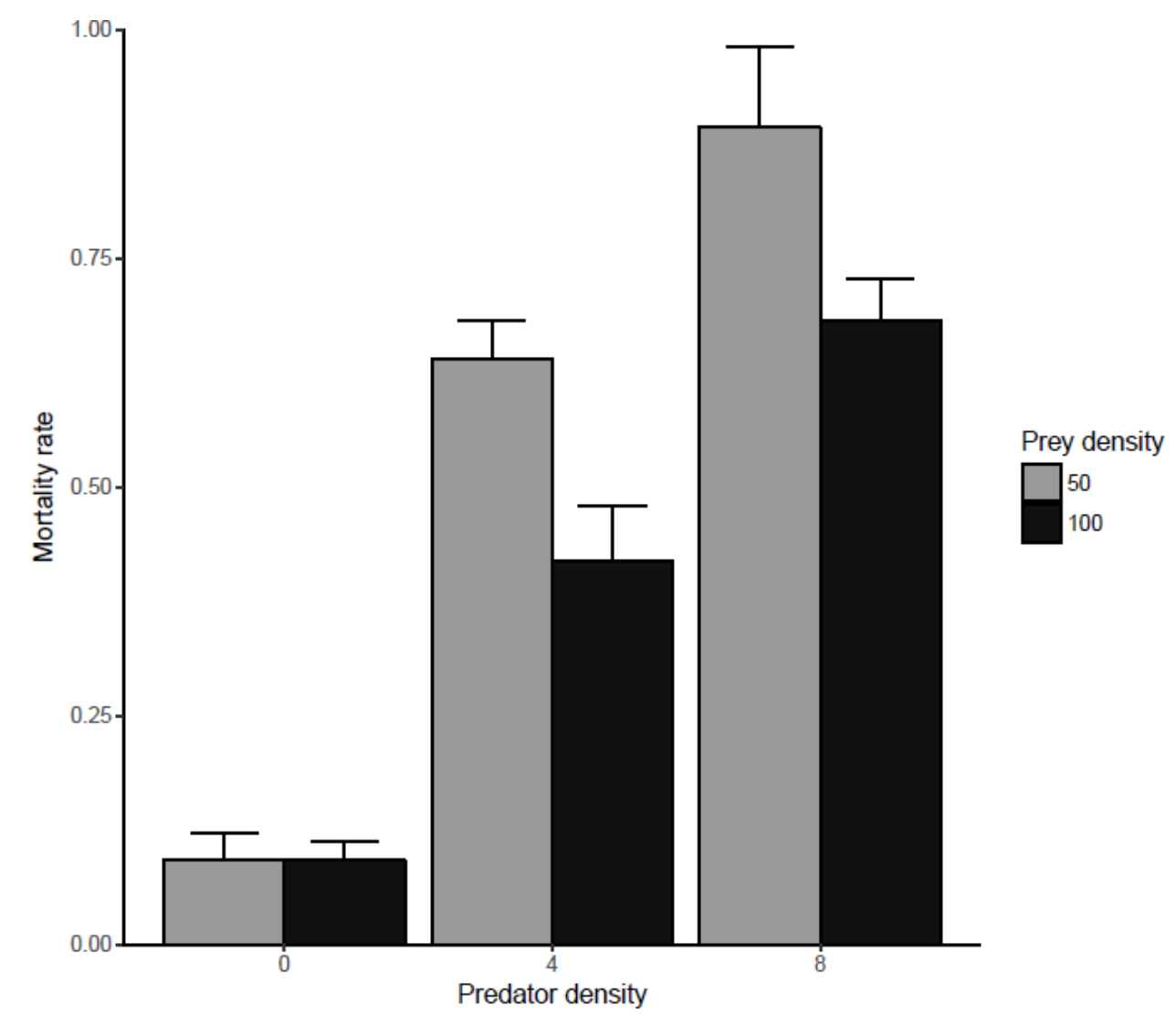

